\title{
Degradation mechanism of SESAMs under intense ultrashort pulses in modelocked VECSELs
}

Sadhvikas Addamane, Darryl Shima, Alexandre Laurain, Hsiu-Ting Chan, Ganesh Balakrishnan, et al.

Sadhvikas Addamane, Darryl Shima, Alexandre Laurain, Hsiu-Ting Chan, Ganesh Balakrishnan, Jerome V. Moloney, "Degradation mechanism of SESAMs under intense ultrashort pulses in modelocked VECSELs," Proc. SPIE 10515, Vertical External Cavity Surface Emitting Lasers (VECSELs) VIII, 105150T (15 February 2018); doi: 10.1117/12.2290684

SPIE. Event: SPIE LASE, 2018, San Francisco, California, United States 


\title{
Degradation mechanism of SESAMs under intense ultrashort pulses in modelocked VECSELS
}

\author{
Sadhvikas Addamane ${ }^{l}$, Darryl Shima ${ }^{1}$, Alexandre Laurain ${ }^{2}$, Hsiu-Ting Chan $^{2}$, Ganesh Balakrishnan $^{1} \&$ \\ Jerome V. Moloney ${ }^{2}$
}

1. Center for High Technology Materials, University of New Mexico, Albuquerque, NM, US

2. College of Optical Sciences, The University of Arizona, Tucson, AZ, US

\begin{abstract}
Mode-locked VECSELs using SESAMs are a relatively less complex and cost-effective alternative to state-of-the-art ultrafast lasers based on solid-state or fiber lasers. VECSELs have seen considerable progress in device performance in terms of pulse width and peak power in the recent years. However, it appears that the combination of high power and short pulses can cause some irreversible damage to the SESAM. The degradation mechanism, which can lead to a reduction of the VECSEL output power over time, is not fully understood and deserves to be investigated and alleviated in order to achieve stable mode-locking over long periods of time. It is particularly important for VECSEL systems meant to be commercialized, needing long term operation with a long product lifetime.

Here, we investigate the performance and robustness of a SESAM-modelocked VECSEL system under intense pulse intensity excitation. The effect of the degradation on the VECSEL performance is investigated using the SESAM in a VECSEL cavity supporting ultrashort pulses, while the degradation mechanism was investigated by exciting the SESAMs with an external femtosecond laser source. The decay of the photoluminescence (PL) and reflectivity under high excitation was monitored and the damaged samples were further analyzed using a thorough Transmission Electron Microscopy (TEM) analysis. It is found that the major contribution to the degradation is the field intensity and that the compositional damage is confined to the DBR region of the SESAM.
\end{abstract}

Keywords: Saturable absorbers, VECSELs, degradation mechanism, TEM.

\section{INTRODUCTION}

Ultrafast lasers - lasers generating short pulses with pulse durations in the pico and femtosecond ranges - have progressed rapidly in the recent years and have made significant impact in the fields of both fundamental science and industrial research. ${ }^{1,2}$ The four key features of ultrafast lasers that have led to their numerous applications are: ultra-short pulse duration, high pulse repetition rate, broad spectrum and high peak power ${ }^{1}$. Ultra-short pulse durations enable highresolution measurements in the time domain and this allows for improved temporal resolution for the observation of highspeed phenomena such as chemical reactions ${ }^{2,3}$, carrier relaxation in semiconductors ${ }^{4}$ and electro-optical sampling of highspeed electronics ${ }^{5,6}$. Lasers with high repetition rates (in the multi-gigahertz range) are essential components in optical interconnects ${ }^{7}$, high-capacity telecommunication systems ${ }^{8}$ and clocks. ${ }^{9}$ In the field of telecommunication, development of a mode-locked laser with a high repetition rate and a tunable emission wavelength around $1.55 \mu \mathrm{m}$ is significant to keep up with the increase in data transmission rates. Pulses with high peak powers focused on nonlinear fibers create a broad optical spectrum, which in turn supports good spatial resolution for techniques like Optical Coherence Tomography ${ }^{10,11}$ (OCT). OCT exploits the fact that mode-locked lasers offer considerably higher average output power when compared to other broadband sources and a broad spatial resolution of a few microns. The enhancement in peak power compared to average power in ultrafast lasers is a consequence of the laser energy being compressed into ultra-short pulse durations. Therefore, mode-locked lasers are capable of producing a record combination of short pulse, high peak power and high average power (Pulse energy $>1 \mu \mathrm{J}$ at $30 \mathrm{MHz}$ repetition rate ${ }^{12,13}$ ). These high peak powers can be used in a variety of applications, from multi-photon absorption technologies ${ }^{14}$ to processing microstructures in solid targets without a corresponding increase in temperature ${ }^{15}$ (non-thermal ablation). In the medical field, these pulses can be used for increased

Send correspondence to S.J. Addamane: Email: addamane@unm.edu

Vertical External Cavity Surface Emitting Lasers (VECSELs) VIII, edited by

Juan L. Chilla, Proc. of SPIE Vol. 10515, 105150T - @ 2018 SPIE

CCC code: $0277-786 \mathrm{X} / 18 / \$ 18 \cdot$ doi: $10.1117 / 12.2290684$

Proc. of SPIE Vol. 10515 105150T-1 
precision in surgical cutting especially for corneal ${ }^{16}$ and brain tumor surgeries ${ }^{17}$. It is to be noted that the description here covers only a few of the numerous applications of ultrafast lasers.

At present, applications requiring stable ultra-short pulses are dominated by Kerrlens mode-locked Ti: sapphire lasers, fiber laser, and directly diode-pumped solid-state lasers (DPSSL) mode-locked using SESAMs ${ }^{18,19}$. These systems have enabled the realization of high average powers ${ }^{20,21}$, pulse energies ${ }^{12,13}$ and repetition rates ${ }^{22}$ with pulses in the femtosecond range. However, despite the high-performance of solid state lasers, they are expensive and invariably have complex designs. On the other hand, semiconductor lasers are cost-effective for mass production and offer a high level of integration to realize compact devices. Furthermore, the characteristics of semiconductor lasers can be easily tailored using band gap engineering. With regard to mode-locking, semiconductor lasers can be classified into edge-emitters and semiconductor disk lasers (SDLs). Edge-emitting mode-locked lasers typically have the same epitaxial layer acting as both the gain and the saturable absorber. While the gain region is essentially a forward biased p-i-n diode, the absorber section is reversebiased. This takes away the ability to optimize both sections independent of each other. On the other hand, it also makes this system extremely compact and enables their direct integration into optical circuits. To date, edge-emitting quantum dot (QDot) lasers at $1550 \mathrm{~nm}$ have been able to achieve pulse durations of $312 \mathrm{fs}$ with a $92 \mathrm{GHz}$ repetition rate and 13.2 $\mathrm{mW}$ of average power ${ }^{23}$. However, in terms of high average power, most mode-locked edge-emitters have been able to operate in the picosecond pulse duration range with only $\sim 250 \mathrm{~mW}$ average power ${ }^{24,25}$. In order to increase the output power of mode-locked edge emitters, external elements ${ }^{26}$ or pulse compression techniques have been employed and this increases the complexity of these systems. Moreover, it is not trivial to increase the output power levels in this system. Edge emitters have a long interaction length and this leads to an increase in the noise levels and limits modelocking stability. It is also important to note that edge-emitters are prone to end facet damage at high peak powers in the watt-levels ${ }^{27}$. In addition, edge-emitting semiconductor lasers inherently have strongly asymmetric beam profiles. SDLs or vertical-external-cavity surface-emitting lasers (VECSELs) are the ideal choice for mode-locking applications as they merge the positive aspects of DPSSLs and edge-emitting semiconductor lasers. Like edge-emitter semiconductor lasers, the design can easily be optimized using band gap engineering. However, unlike edge-emitters, the optical field in a VECSEL has a short interaction length with the gain medium and circulate in a high Q cavity, which reduces the noise level and makes it easier to achieve higher output powers with a symmetric circular beam profile. On the other hand, power scaling in VECSELs can be achieved by simply increasing the mode size (very similar to DPSSLs). Also, like DPSSLs, a VECSEL chip coupled with a SESAM can be used to achieve a self-starting, stable mode-locking state. Moreover, VECSELs open up the possibility of integrating the SESAM within the gain region which is referred to as a mode-locked-integrated external cavity surface emitting laser. (MIXSEL)

\subsection{Motivation}

Although SESAM-modelocked VECSELs have proven to be a suitable choice for modelocking applications, there is still a lot of room for improvement for commercializing this system. As a result of being exposed to high peak powers, SESAM chips in this system suffer degradation of output power over time. The mechanism for this decay in output power over time and its physical implications to the epitaxial layers of the SESAM chip is not yet fully understood. This needs to be investigated and alleviated in order to achieve stable mode-locking over long periods of time. Therefore, this work focuses on studying and understanding the degradation mechanism in SESAM mode-locked VECSEL systems in order to be able to increase modelocking lifetime for long-term operation.

For this study, we have chosen to use the well-established anti-resonant InGaAs QW-based SESAM system around the $1 \mu \mathrm{m}$ wavelength range. A standard VECSEL structure (emission wavelength of $1030 \mathrm{~nm}$ ) with an AlGaAs-GaAs DBR and an active region with multiple InGaAs quantum wells placed non uniformly in GaAsP barriers is used. The VECSEL structure optimization was not part of this work. The SESAMs consist of 29-pairs of GaAs/AlAs for the DBR region, InGaAs quantum well $(\mathrm{QW})$ as the absorber and GaAs spacer and cap layers. While the thicknesses for the DBR layers and the QW are optimized for 1030nm, the thicknesses of the spacer and cap layers are varied as part of this study.

\section{EXPERIMENTAL SETUP}

The SESAM structures investigated here are grown using Molecular Beam Epitaxy (MBE) in a VG V80H Semicon reactor on GaAs (001) substrates. Prior to epitaxial growth, the native oxide on the GaAs substrate is removed by thermal desorption of the substrate at $\sim 620^{\circ} \mathrm{C}$ for $20 \mathrm{~min}$ under an arsenic $\left(\mathrm{As}_{2}\right)$ overpressure. This is followed by the growth of a $200 \mathrm{~nm}$ thick GaAs smoothing layer at $580^{\circ} \mathrm{C}$. Once the GaAs is smoothened out, the growth of the DBR layers consisting 
of $0.25 \lambda$ thick AlAs/GaAs is initiated. For a design wavelength of $1030 \mathrm{~nm}$, the GaAs and AlAs thicknesses turn out to be 88.2 and $74.4 \mathrm{~nm}$ respectively. Although 24-25 pairs would yield sufficient reflectivity, for the SESAMs described in this work, we have used a 29-pair DBR to minimize the non-saturable losses. Following this, a GaAs spacer layer is grown at the DBR growth temperature $\left(580^{\circ} \mathrm{C}\right)$. The substrate temperature is then brought down to $475^{\circ} \mathrm{C}$ for the growth of the QW. The composition and thickness of the QW are $\mathrm{In}_{0.18} \mathrm{Ga}_{0.82} \mathrm{As}$ and $8.35 \mathrm{~nm}$ respectively. After the growth of the $\mathrm{QW}, \mathrm{a} 1.5 \mathrm{~nm}$ thick cold GaAs cap is grown to protect the QW. For the growth of the rest of the GaAs cap, the substrate temperature is brought back up to $580^{\circ} \mathrm{C}$. The growth rates of In and Ga were $0.06 \mathrm{ML} / \mathrm{sec}$ and $0.3 \mathrm{ML} / \mathrm{sec}$ respectively (in a ratio of $\mathrm{r}_{\mathrm{In}} /$ $\mathrm{r}_{\mathrm{Ga}}=0.18 / 0.82$ for the composition of the $\mathrm{QW}$ ) and a constant As: Ga ratio of 13 is maintained. After the growth, the semiconductor structure is coated with a dielectric coating of $\mathrm{Ta}_{2} \mathrm{O}_{5}$ with thicknesses optimized to obtain a spectrally flat group delay dispersion (GDD).

The VECSEL and SESAM are tested in a ring cavity configuration. The VECSEL chip (gain wafer) is placed at 1/4 of the total cavity length $L=92 \mathrm{~mm}$ from the SESAM. This offers symmetrical amplification of the two pulses traveling in the two different directions and makes sure that the pumping duration and gain recovery are equal for both pulses. A highly reflective concave mirror with $r=50 \mathrm{~mm}$ and a flat output coupler with a reflectivity of $99.2 \%$ complete the cavity. This design provides a mode radius of $127 \mu \mathrm{m}$ on the gain chip and $67 \mu \mathrm{m}$ on the saturable absorber. The angle of incidence on both the VECSEL and the SESAM is adjusted to $\sim 7^{\circ}$.

In order to quantify and optimize the lifetime of SESAMs and study the effect of various cavity conditions such as the incident fluence and the dielectric coating, the output power stability measurement from the SESAM would have to be repeated on a number of samples. This is inherently time-consuming as it takes a substantial amount of time to optimize the mode-locking state in the cavity for each sample. Therefore, we have used a pulsed PL experiment using femtosecond pulses ( 100fs@ 780nm) from a mode- locked laser to characterize the damage mechanism on various samples in a timely manner. Moreover, this setup offers the flexibility of easily changing the incident fluence on the SESAMs to find the damage threshold for each sample. It also closely simulates the conditions for the SESAM in a mode-locked state in the femtosecond regime. For these measurements, we define "damage" of a SESAM as an irreversible decrease in the PL intensity over time. It is to be noted that a decrease in PL intensity over time does not necessarily imply a damage to the saturable absorber (QW here) as the PL is heavily modulated by the DBR layers and this contributes to the PL intensity. The incident fluence is measured using a power meter before the measurement is started and changed when required by controlling the driver current to the laser. Once the pulses are incident on the sample, the PL measurement is repeated every $30 \mathrm{sec}$ (each measurement takes $\sim 1 \mathrm{sec}$ ) over $5 \mathrm{~min}$ and each measurement is recorded to analyze the effect on PL intensity. Once a spot on the sample is damaged, the SESAM stage is moved either in the $\mathrm{x}$ - or the $\mathrm{y}$-direction to an undamaged spot for the next measurement.

For the TEM analysis, the damaged and as-grown SESAMs are cross-sectioned with a focused ion beam (FIB) lift out using a FEI Helios 450 DIB system. The TEM images were taken using an FEI Tecnai F20 equipped with HAADF STEM detector.

\section{RESULTS}

For baseline modelocking results, the SESAM structure described in the previous section is grown and tested in a ring cavity. The spacer and cap layers are kept at $22 \mathrm{~nm}$ and $5 \mathrm{~nm}$ respectively for the reference sample.

\subsection{Reference modelocking results}

Figure 1(a) below shows the room-temperature reflectivity spectrum for the reference SESAM. The DBR stop-band extends from $975 \mathrm{~nm}$ to $1080 \mathrm{~nm}$ and is centered at $\sim 1020 \mathrm{~nm}$. Although this is slightly blueshifted $(\sim 1 \%)$, the reflectivity is sufficiently high at $1030 \mathrm{~nm}$. The dip in reflectivity at $\sim 1025 \mathrm{~nm}$ can be attributed to absorption from the InGaAs QW. Based on the reflectivity spectra, the anti-reflection coating $\left(\mathrm{Ta}_{2} \mathrm{O}_{5}\right)$ is designed and deposited to reduce the third order dispersion and enhance absorption. The results discussed from this point onwards for the rest of this section refer to coated QW SESAMs. The GDD for the QW-based SESAM around the wavelength of interest (1030 $\mathrm{nm})$ is measured before further device testing. The anti-reflection coating reduces the cavity enhancement and flattens the GDD profile as expected. As figure 1(b) shows, the GDD is relatively flat between $1020-1030 \mathrm{~nm}$ and stays positive. 


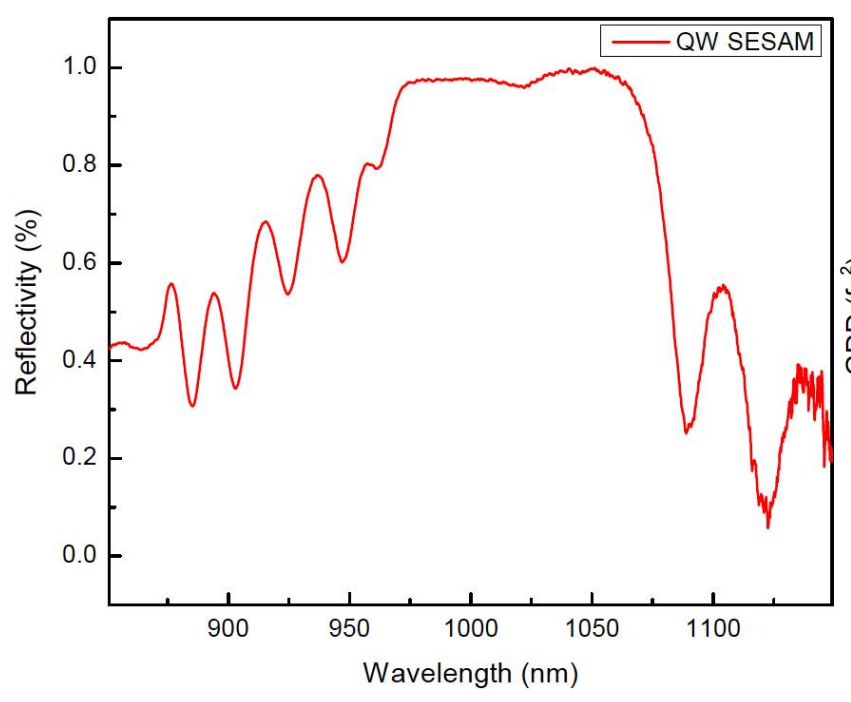

(a)

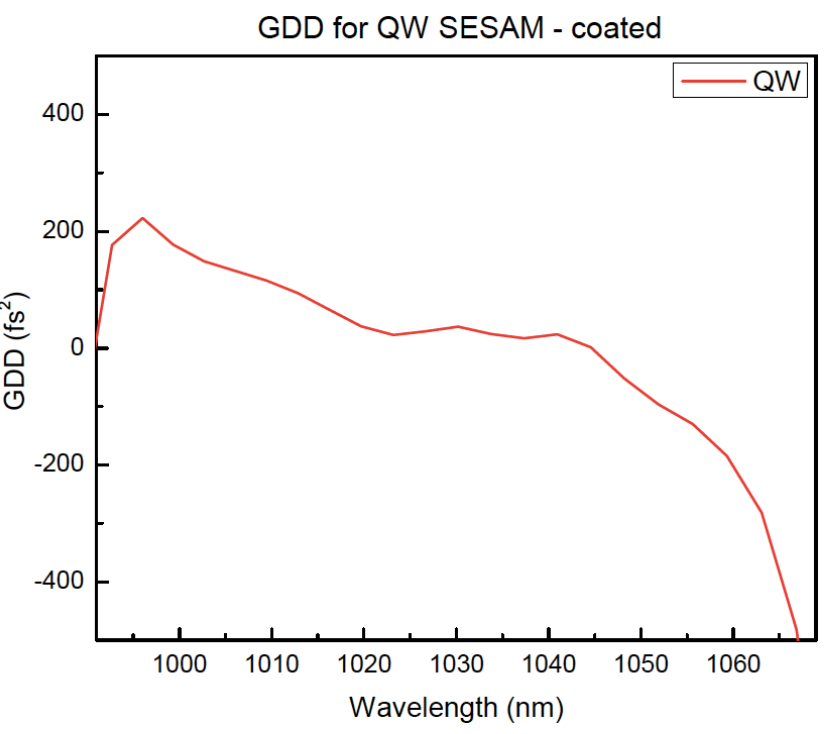

(b)

Figure 1 (a) : Room-temperature reflectivity spectrum from InGaAs QW-based SESAM with the stopband wavelength centered at $\sim 1020 \mathrm{~nm}$ (b) GDD profile for a QW-based SESAM around the absorption wavelength

With the different parameters for the SESAM (GDD, reflectivity etc.) verified to be able to support ultra-short pulses, we attempted mode-locking the SESAM in an asymmetric ring cavity (as explained in the previous section). A minimum pulse duration of $128 \mathrm{fs}$ (non-collinear SHG autocorrelation trace of the output fitted to a $128 \mathrm{fs} \operatorname{sech}^{2}$ pulse shape) was obtained with the gain element at $25^{\circ} \mathrm{C}$ and the SESAM chip at $55^{\circ} \mathrm{C}$ with an output power of $90 \mathrm{~mW}$ per output beam for a pump power of $22 \mathrm{~W}^{28}$.

\subsection{Operation lifetime of QW-based SESAMs}

As described in the previous section, we were able to successfully realize the growth and characterization of InGaAs QWbased SESAMs able to support pulses as short as $128 \mathrm{fs}$. Although this is close to the shortest pulse durations realized from a mode-locked VECSEL system, for this system to be commercially relevant in high-power technologies using SESAMs, the temporal stability of the mode-locked state must be guaranteed. For the sustainability of short pulses in the femtosecond regime, a robust SESAM design that can tolerate high intracavity peak powers is required. Moreover, the power scalability of thin-disk lasers by increasing the spot size on the gain region places more restrictions on the SESAM design. Therefore, it is critical that SESAMs that can support ultra-short pulse durations also have long operating lifetimes and low nonsaturable losses to avoid thermal effects. This section focuses on quantifying the mode-locking operating lifetime of InGaAs QW-based SESAMs in the femtosecond regime.

\subsubsection{Degradation of output power in QW-based SESAMs}

Although the SESAMs characterized in the previous section were able to support some of the shortest pulse durations reported to date, it is found that they exhibit poor power stabilities with effective mode-locking lifetimes $<30$ min. Figure 2 shows the gradual degradation of an InGaAs-QW based SESAM mode-locked with a $128 \mathrm{fs}$ pulse in a symmetric ring cavity. The average output power decreases continuously for $\sim 30 \mathrm{~min}$ before the lasing state is lost altogether. It is interesting to note here that this decay in output power does not merely drive the cavity out of the mode-locking state to a continuous-wave (CW) state, but rather the VECSEL chip entirely stops lasing. Also, the degradation is specific to pulses with shorter durations ( $<500 \mathrm{fs}$ ) and does not occur when the pulse duration is longer (even with higher average powers). Another interesting aspect is that when this process occurs, the decay in output power is always accompanied by a visible green emission from the SESAM chip. The initial rate of degradation in the output power can be calculated to be $6.5 \% / \mathrm{min}$ which gradually decreases to $0.8 \%$ after $10 \mathrm{~min}$. These are rather high degradation rates and the effective lifetime is substantially shorter than required for commercial technologies. Therefore, it is critical to understand the decay mechanism in play here and potentially propose a solution to increase SESAM lifetimes. Before performing any further studies to 
determine the decay mechanism, we decided to investigate any past work that has been done towards solving this issue.

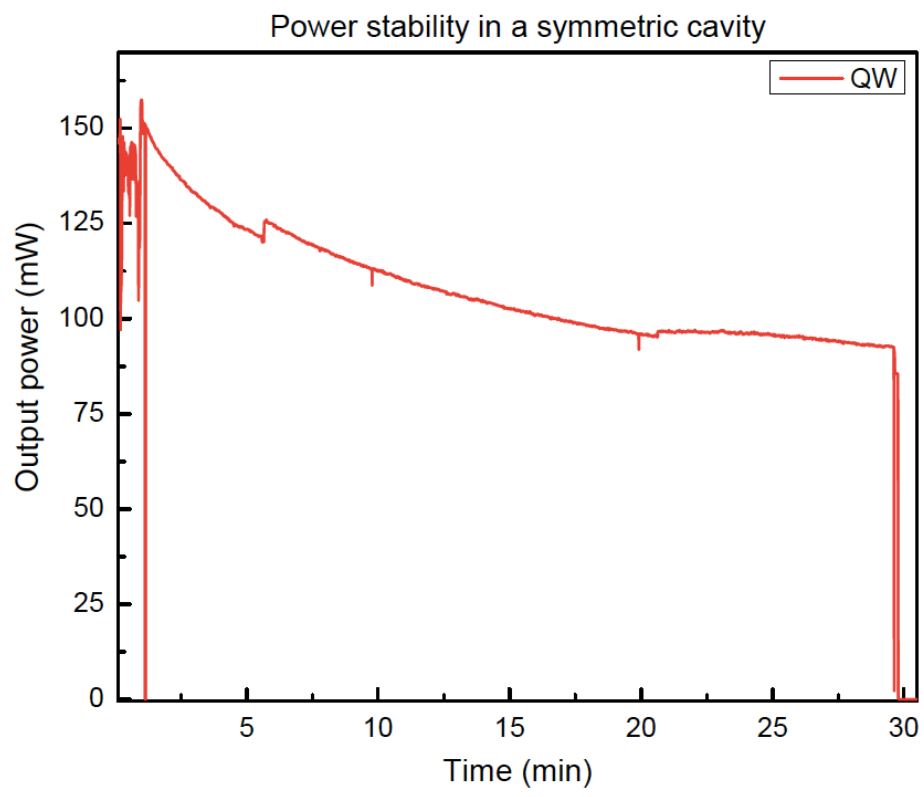

Figure 2: Output power stability for a InGaAs QW-based SESAM mode-locked with a $128 \mathrm{fs}$ pulse in a ring cavity

\subsection{Possible mechanism causing SESAM degradation}

As the use of mode-locked systems has become more technologically viable in the recent years, there has been an increased interest in studying degradation and damage thresholds in SESAMs. ${ }^{29-36}$ Among all the studies carried out to measure the damage threshold of InGaAs QW-based SESAMs, it is fairly widely accepted that the damage mechanism is dominated by two-photon absorption (TPA), especially in the case of femtosecond pulses. TPA is a non-linear process by which a carrier is excited by two photons ${ }^{37}$, the sum of whose energies corresponds to a very high-energy state in the conduction band of the semiconductor. This phenomenon occurs with significant rates only at high optical intensities- for instance in mode-locked systems with femtosecond pulses - because the absorption coefficient is proportional to the optical intensity.

The theory of TPA causing the damage certainly explains the observation of visible green emission from the SESAMs during the process of degradation. However, although most studies on SESAM damage agree on the mechanism being TPA, there is still a debate on a few other aspects of the degradation process. While some results show TPA is more dominant in the DBR region ${ }^{29,30}$, some report that it is the QW that is being affected ${ }^{33}$. Moreover, regardless of the location of damage, there hasn't been a comprehensive study conducted to investigate the physical damage that TPA causes on the epitaxial layers. Therefore, the rest of this work focuses on studying the effect of different SESAM parameters on the damage threshold and examining the physical damage caused by TPA.

\subsubsection{Dependence on incident fluence}

Figure 3 shows the PL degradation of an InGaAs-QW based SESAM (same structure used as in output power stability measurements) while the structure was being pumped with a modelocked laser producing $100 \mathrm{fs}$ pulse duration at $780 \mathrm{~nm}$ with a repetition rate of $80 \mathrm{MHz}$ and was focused on a spot size of $50 \mathrm{um}$. The average power was varied from $10 \mathrm{~mW}$ to $50 \mathrm{~mW}$ and $90 \mathrm{~mW}$. The degradation is very slow with an incident power of $10 \mathrm{~mW}$ but as the power was increased to $\sim 30 \mathrm{~mW}$, there was substantial PL degradation ( $\sim 10 \%$ of original intensity after $1 \mathrm{~min})$ between each measurement. It is evident from the measurements at 50 and $90 \mathrm{~mW}$ that the PL intensity decay is faster with higher incident fluence. Also, it is interesting to note that the trend in degradation is not linear and slows down over time. This is identical to the trend in output power decay as seen in the previous measurement in a mode-locked cavity. 

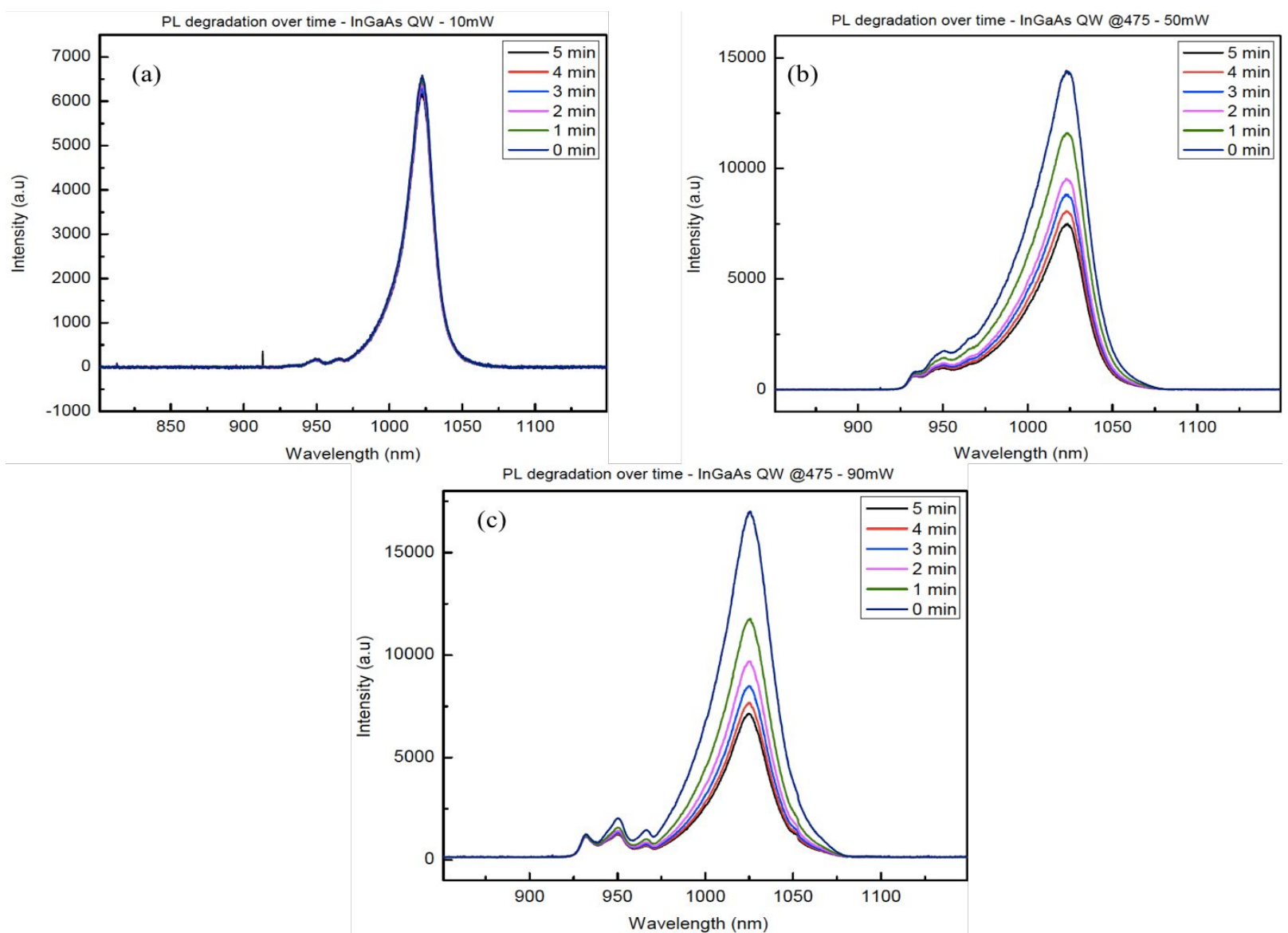

Figure 3: Degradation in PL intensity for InGaAs QW-based SESAMs for different incident power - 10mW (a); 50mW (b) ; 90mW (c)

In order quantify the decay in PL intensity observed in the previous figure, the PL peak intensities for each measurement are determined, normalized to the original intensity (intensity at $\mathrm{t}=0 \mathrm{~min}$ ) and plotted as a function of time in figure 4 .

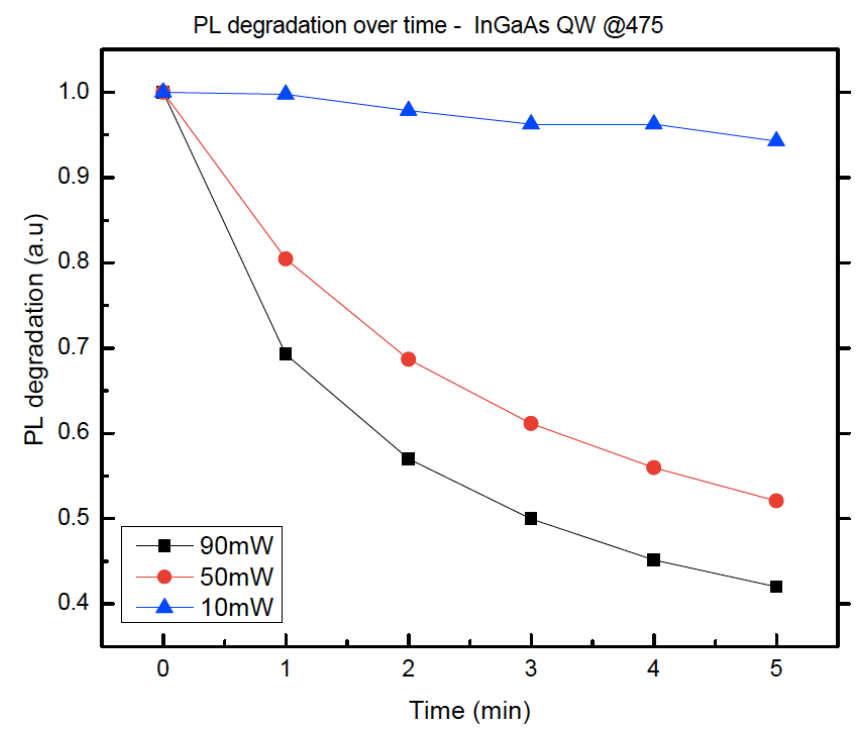

Figure 4: Degradation in PL peak intensities (normalized to the first measurement) over time for different incident fluences. 
The decay in PL peak intensities can be fitted (not shown here) to an exponential decay function (with an offset)

$$
y=y_{0}+A_{1} e^{-\frac{\left(x-x_{0}\right)}{\tau}}
$$

where $\mathrm{y}_{0}$ is the $\mathrm{y}$-offset ; $\mathrm{x}_{0}$ is the center; $\mathrm{A}_{1}$ is the amplitude and $\tau$ is the decay time constant. The relevant parameter for the PL degradation is $\tau$ which would be the time taken for the PL peak intensity to reduce to $1 / \mathrm{e}(36.8 \%)$ of the original value. At $10 \mathrm{~mW}$, the trend in degradation is not a good fit for the equation above. However, the time constants $(\tau)$ for incident powers of $50 \mathrm{~mW}$ and $90 \mathrm{~mW}$ can be shown to be $2.27 \mathrm{~min}$ and $1.47 \mathrm{~min}$ respectively. Therefore, the rate of degradation increases by a factor of $\sim 1.5$ for an increase in incident fluence from 50 to $90 \mathrm{~mW}$.

\subsubsection{Dependence on cap thickness}

At this point in the study, we suspected that the PL decay and consequently, the decay in SESAM output power was based on degradation of the InGaAs QW by oxidation, due to its close proximity to the semiconductor-air interface. This mechanism has been known to cause catastrophic optical damage in InGaAs QW lasers through facet oxidation or defect formation under high-power operation ${ }^{38-41}$. To test this hypothesis, we wanted to examine if the rate of degradation would depend on the proximity of the InGaAs QW to the semiconductor-air interface. Therefore, we performed the PL decay test with 3 different SESAM structures with identical GaAs/AlAs DBRs, 22nm GaAs spacer layers and InGaAs QW absorber but varying cap thicknesses. ( $3 \mathrm{~nm}, 5 \mathrm{~nm}$ and $7 \mathrm{~nm}$ ). Figure 5 shows a summary from this measurement. The three plots (a), (b) and (c) show the PL decay for three incident powers (20,30 and 50mW) and within each plot, the cap thickness is varied between $3 \mathrm{~nm}, 5 \mathrm{~nm}$ and $7 \mathrm{~nm}$. As in the previous experiment, the normalized PL peak intensity is tracked over time for different cap thicknesses and different incident power. In general, the trend in the PL decay shows that the degradation rate indeed increases with decreasing cap thickness and increasing incident fluence. This supports our hypothesis that the damage mechanism is related to the distance between the InGaAs QW and the air-semiconductor interface.

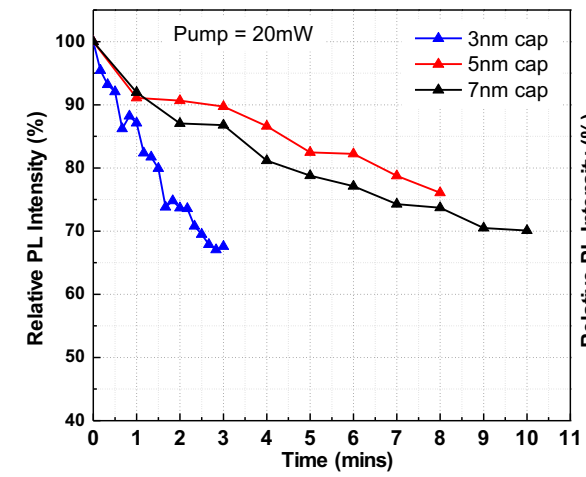

(a)

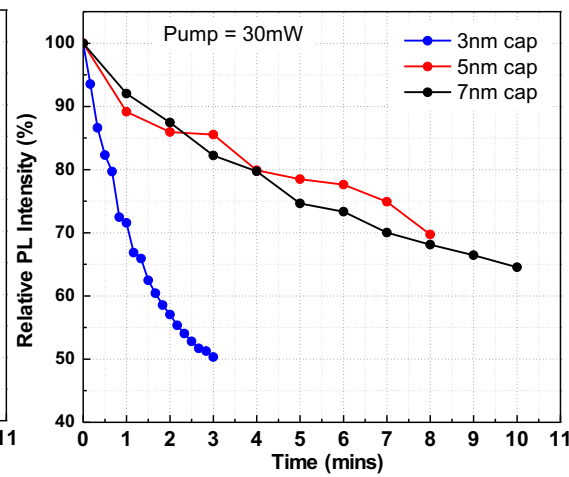

(b)

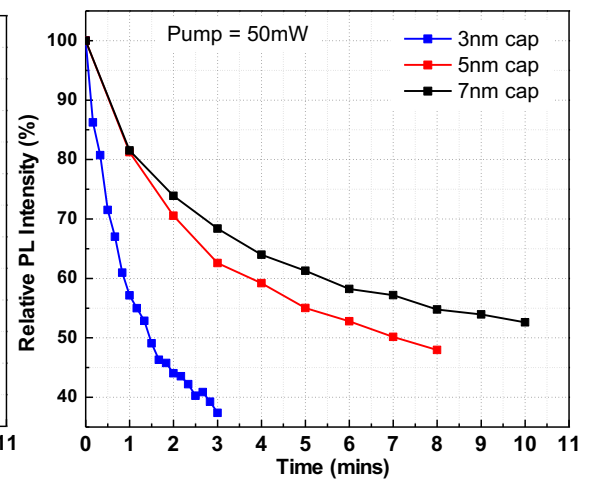

(c)

Figure 5: PL decay test is performed on InGaAs QW-based SESAMs with different GaAs cap thicknesses ( $3 \mathrm{~nm}$, 5nm and 7nm). Row (1) shows 3 plots $(a, b, c)$, each for a specific cap thickness, and the incident fluence is varied within each plot. Row (2) shows 3 plots $(a, b, c)$ each for a specific incident fluence and all three caps thicknesses are tested with the same incident fluence.

In order to quantify the effect of the GaAs cap thickness on the rate of degradation, we chose the results for the three different cap thicknesses $(3,5$ and $7 \mathrm{~nm}$ ) with the highest incident fluence of 50mW (shown in Fig.5 (c) above). This data is fitted to the exponential decay function (Equation 1) to compute the dependence of the decay time constant $(\tau)$ on the cap thickness. The exponential decay fit is shown in figure 6 for a GaAs cap thickness of $3 \mathrm{~nm}$ (a), $5 \mathrm{~nm}(\mathrm{~b})$ and $7 \mathrm{~nm}(\mathrm{c})$. From the fitting parameters, the decay time constant $(\tau)$ can be calculated to be $0.927,2.536$ and 2.682 min for 3,5 and $7 \mathrm{~nm}$ caps respectively. From this, it is evident that the rate of degradation decreases as the distance between the absorber and the semiconductor-air interface increases. It is also interesting to note that the decrease in the decay time constant with decreasing cap thickness is not linear but rather exponential in nature. 

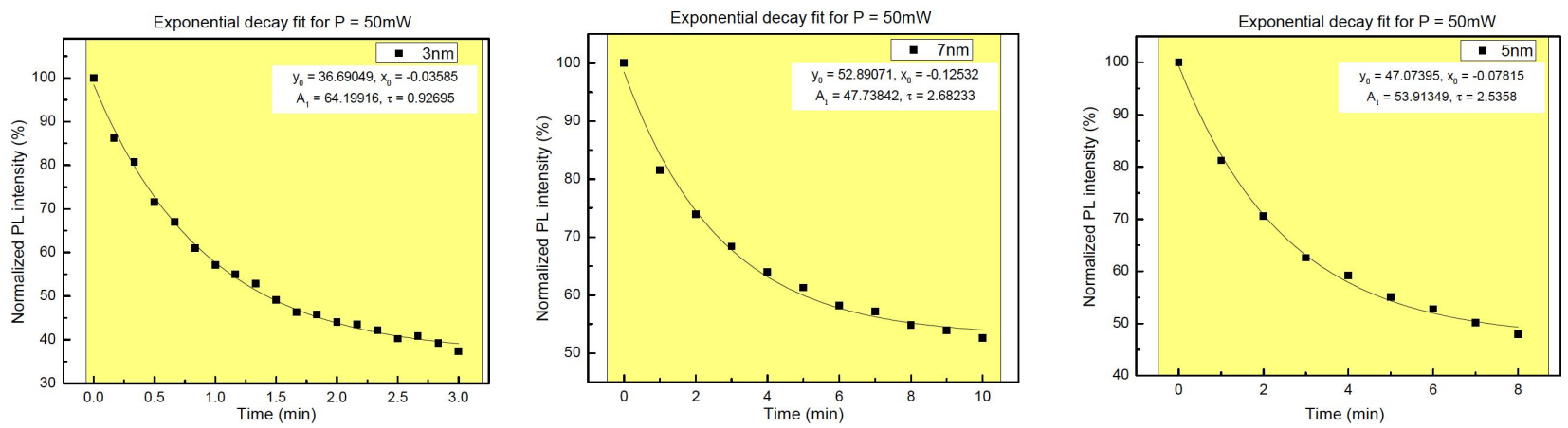

Figure 6: Exponential decay (with offset) fit for InGaAs QW-based SESAMs with varying cap thickness $-3 \mathrm{~nm}(\mathrm{a}) ; 5 \mathrm{~nm}(\mathrm{~b})$ and $7 \mathrm{~nm}(\mathrm{c})$

\subsubsection{Dependence on dielectric coating.}

The effect of the GaAs cap thickness on the degradation rate of SESAMs (as shown in the study above) certainly seems to support the theory that SESAM degradation is related to a certain extent to damage caused to the absorber layer. Assuming that high fluence causes oxidation of the absorber, it becomes critical to understand the effect of the dielectric coating $\left(\mathrm{Ta}_{2} \mathrm{O}_{5}\right)$ that is deposited to help flatten the GDD profile on the degradation of the SESAM. The presence of an oxide layer close to the QW might potentially provide more or less access to oxygen and consequently modify the degradation rate. In order to confirm this, the PL degradation test is performed on coated and uncoated InGaAs-QW based SESAMs. To avoid the effects of growth drifts, the devices chosen for this study are from the same wafer and both devices were located at identical distances from the center of the wafer.

Figure 7 shows the decay in PL peak intensity at a power of $90 \mathrm{~mW}$ for both coated and uncoated SESAM samples. The peak intensity values are again normalized to the peak intensity at $0 \mathrm{~min}$. It is found that the rate of degradation is higher with the coated SESAMs when compared to the uncoated SESAMs. From the slopes of the lines connecting each measurement, a small difference can be observed in the initial degradation rate between the two samples. When fit to a exponential decay function (refer to equation (1) above), the decay constant $(\tau)$ is found to decrease from $1.47 \mathrm{~min}$ for a uncoated sample to $0.85 \mathrm{~min}$ for a coated one. This result also seemed to substantiate the claim that the degradation of SESAMs might be caused by a damage (possibly oxidation) of the QW. It must be noted however that the change of enhancement factor between coated and uncoated sample could also increase the amount of absorption and TPA and thus explain the decay rate difference.

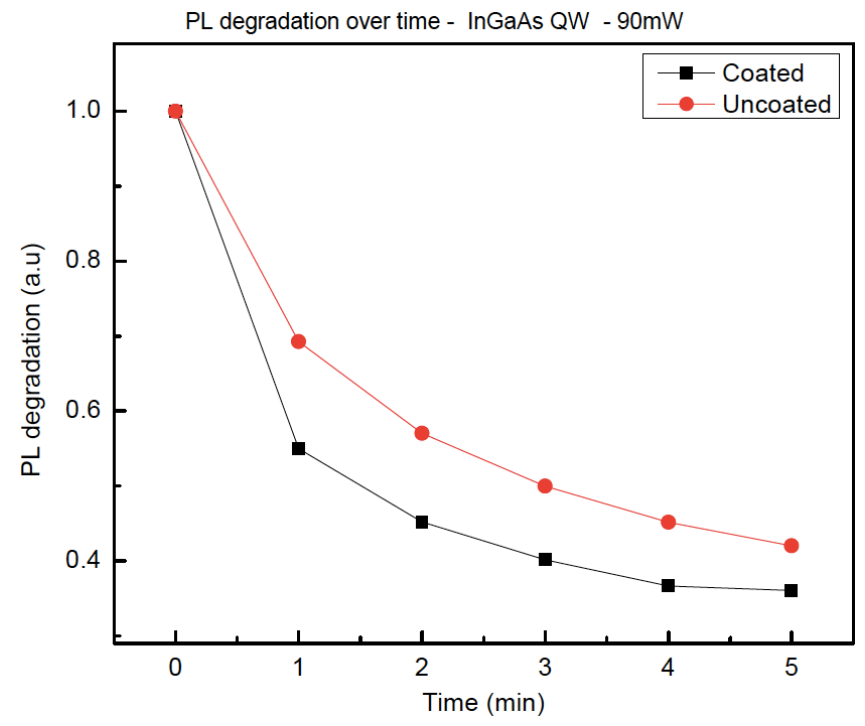

Figure 7: PL peak intensity degradation over time compared between coated and uncoated samples. The degradation rate is visibly higher for the coated device. 


\subsection{TEM analysis}

Damage threshold measurements described earlier in this section based on incident fluence, cap thickness and presence of dielectric coating layer seem to suggest that the damage mechanism is related to degradation of the absorber. In order to confirm this hypothesis and to observe the damage to the epitaxial QW layer, we have performed a cross-sectional transmission electron microscopy (TEM) analysis of "damaged" versus as-grown samples. The "damaged" devices are standard InGaAs QW-based SESAMs described in the previous chapter (29.5 pair AlAs-GaAs DBR/22nm GaAs spacer $/ 7 \mathrm{~nm}$ GaAs cap) used in a ring cavity to mode-lock a 1030nm VECSEL with $\sim 100 \mathrm{fs}$ pulses for long periods of time. Once a certain spot on the sample is degraded i.e. the output power decays and the mode-locking state is unrecoverable, the sample is moved in the $\mathrm{x}$ - or $\mathrm{y}$ - direction to a different spot. This process is repeated until the entire area of the sample is used. This ensures that any random cross-section of the sample observed would show the effects of the damage mechanism.

Figure 8 shows a comparison of cross-sectional TEM images of the as-grown and damaged SESAMs. The DBR and absorber layers are appropriately labeled on the figure. At this lower magnification, the effects of the damage are seen mainly in the DBR section. Within the DBR itself, particularly the top-section of the GaAs/AlAs layers (close to the airsemiconductor interface) appear damaged. It is interesting to observe that the overall damage profile seen in (b) appears similar to laser-induced damage and resembles a typical laser-beam profile. However, since the absorber layers (GaAs spacer/InGaAs QW/GaAs cap) are not visible, we cannot comment on any degradation in these layers at this point.

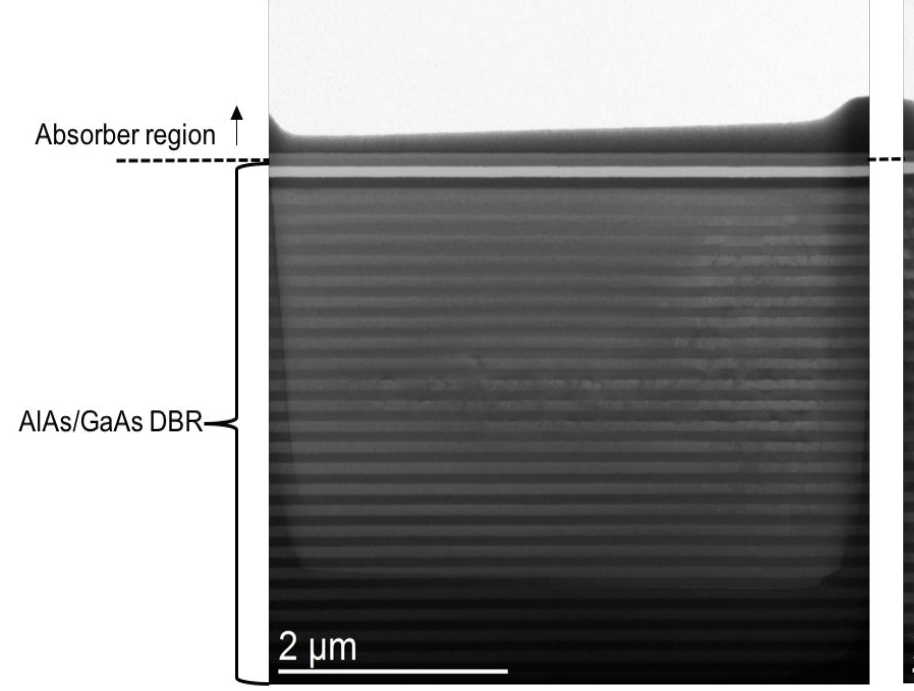

(a)

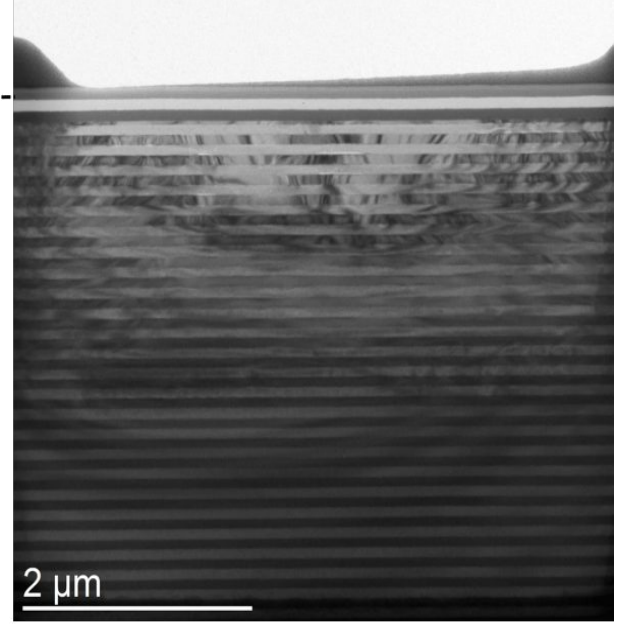

(b)

Figure 8: Cross-sectional TEM images of as-grown and "damaged" SESAMs. The degradation observed in the damaged SESAM is confined to the top DBR layers

In order to study the damage profile in more detail, TEM images at a higher magnification focusing on the top-section of the DBR and the absorber layers are analyzed. Although the absorber region is still not resolved, the last few pairs of the DBR are clearly disfigured and the extent of damage decreases going away from the air-semiconductor interface in figure 9. At the outset, it seems like the damage could be confined to one of the layers of the DBR. However, this would have to be confirmed by a comprehensive elemental analysis using EDS for both layers. Using higher magnification images, the extent of damage, if any, in the absorber region and the damage profile are studied in more detail. 


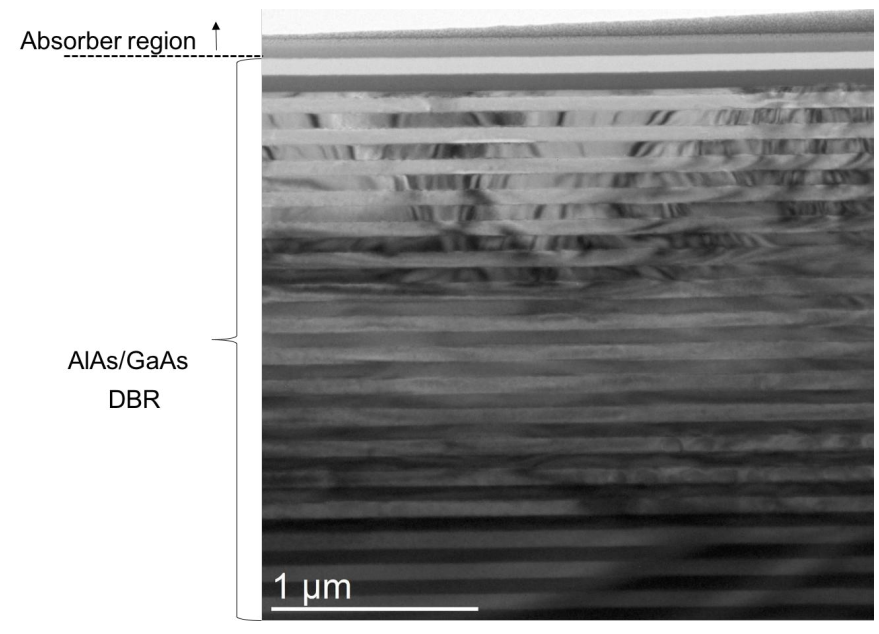

Figure 10: Cross-sectional TEM image at high-magnification showing "damaged" SESAM. The extend of degradation in the DBR layers decreases going away from the semiconductor-air interface.

Figure 11 below shows DF-STEM images of the absorber region (spacer, QW and cap) with the topmost GaAs/AlAs pair of the 29.5 pair DBR for both the as-grown and the damaged SESAM. Contrary to our previous assumptions, the absorber region, including the InGaAs QW is unaffected by the SESAM degradation and seems to be in pristine condition. It is also interesting to note that the GaAs layers on either sides of the QW appear to be free of any damage. On the other hand, the damaged SESAM distinctly shows some material degradation in the final AlAs layer of the DBR.

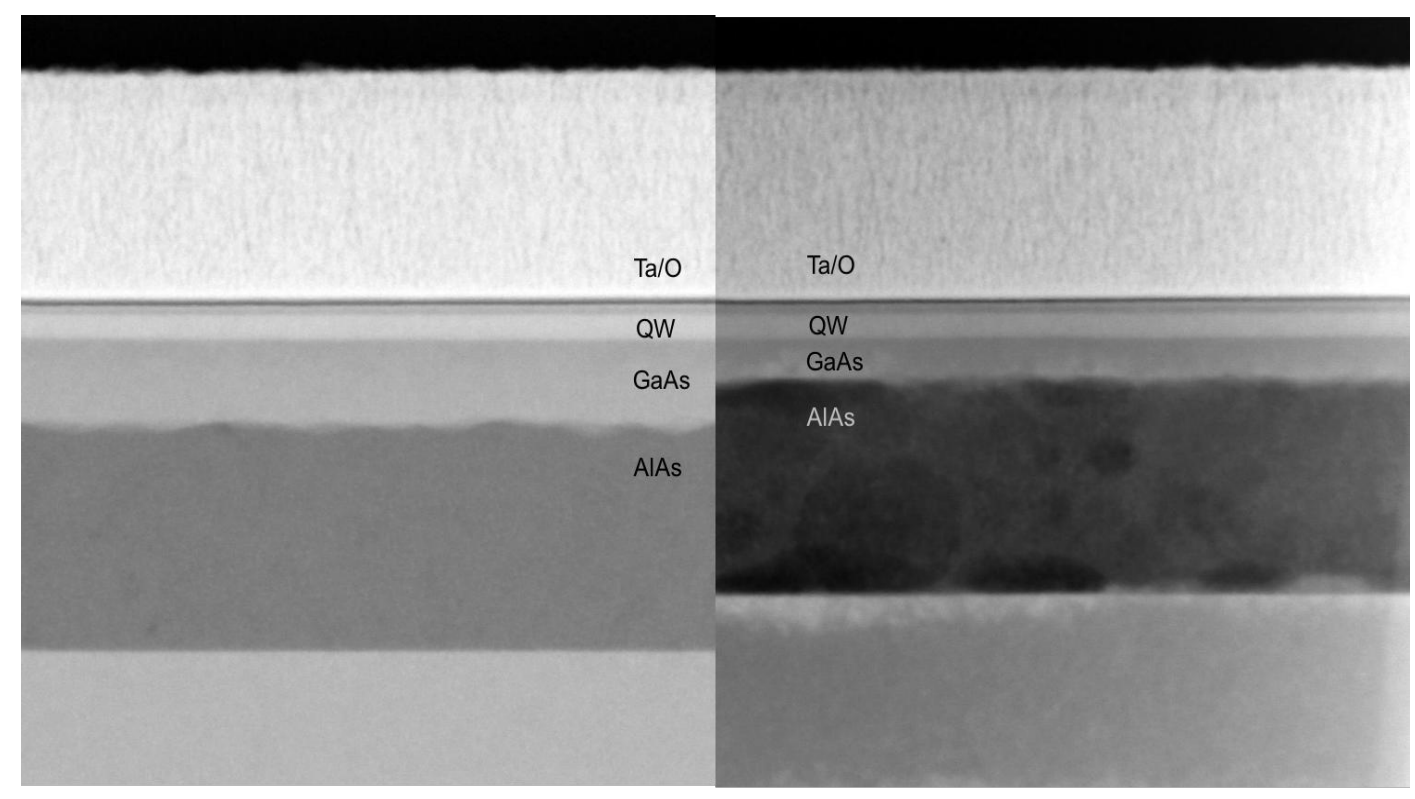

(a)

(b)

Figure 11: DF-STEM images of the absorber region and last DBR-pair of the as-grown (a) and damaged (b) SESAM. While the absorber region seems unaffected, distinct damage is observed in the AlAs region of the damaged SESAM. 


\section{DISCUSSION}

Assuming TPA as the dominant mechanism for SESAM degradation, some questions remained to be answered in order to improve the operating lifetime of these devices. The first question to be answered was, how is the damage affecting the laser performance? The measurements of the PL decay and output power decrease over time for different type of structure and incident power was carried out to answer this question and to quantify the device lifetime and damage threshold. From this study, it is clear that the high field intensity encountered with ultrashort pulses leads to catastrophic and unrecoverable damage of the SESAM, leading to a power decrease and eventually a shut off the laser altogether or makes it hop into a CW state. No clear damage "threshold" was observed but our studies clearly show a gradual degradation over time with an exponential trend, with a time constant that is decreasing with a higher incident power. Also, as mentioned earlier, the degradation process only occurs with ultra-short pulse durations $(<300 \mathrm{fs})$ and does not occur with longer pulse durations even with higher average powers. These two factors seem to suggest that the damage mechanism is related more to the field intensity than the incident average power (and consequently, thermal effects). This relation between the damage threshold and the field intensity explains the dependence of the SESAM degradation on incident fluence, cap thickness and the AR coating. In order to understand their effect on degradation, we studied the effect of varying these three parameters on the field strength in the DBR region. On closer investigation, it is found that the increase in fluence, decrease in cap thickness and the presence of the AR coating effectively increase the field intensity in the DBR region, thus accelerating the SESAM damage.

The second question to be answered was what part of the semiconductor structure is being damaged (coating, QW, barriers, DBR) and to what extent is it being damaged? From the TEM results, it is found that the damage profile closely resembles a laser beam-induced damage. It also shows that the layers being visibly damaged are the AlAs layers of the DBR close the QW, i.e., where the field intensity is the most significant. Assuming TPA is the dominant mechanism, the effect of TPA on any material can be quantified based on its TPA coefficient $\beta$. Higher the value of $\beta$, higher the effect of TPA on any given material ${ }^{29}$. The TPA co-efficient, in turn, is a function of the band gap of the material, and materials with higher band gap energies are less susceptible to TPA ${ }^{42}$. Based on this, the effect of TPA should be considerably higher in the GaAs layer than in the AlAs or the dielectric coating layers ${ }^{29,33}$. However, the TEM images show that the damage might be initiated in the AlAs layer. To explain this, we suspect that the effect of TPA in the GaAs layer causes lattice heating which, in turn, leads to the damage of the AlAs layer.

\section{CONCLUSIONS}

Using a InGaAs QW-based SESAM, we were able to achieve mode-locking with one of the shortest pulse durations reported in literature. However, we also found that these SESAMs exhibit poor temporal stability with effective modelocking lifetimes $<30 \mathrm{~min}$. Through a systematic PL study, we studied the effect of various SESAM parameters such as cap thickness, presence of AR coating and incident fluence on the damage threshold of the SESAM. TPA is recognized to be the damage mechanism which causes a decay in output power over time. In order to observe the damage caused by TPA to the epitaxial layers, we also conducted a thorough TEM analysis of the damaged SESAM compared to an "as-grown" SESAM. Through the TEM study, we were able to conclude that the SESAM degradation is mainly caused by damage in the AlAs layer in the DBR. The damage profile resembles the profile of a laser beam-induced damage with the top of the DBR being more affected than the bottom. These experiments suggest that the damage in the DBR region is the main cause of power degradation and is directly related not to the average power but to the field intensity of the standing electric field.

\section{REFERENCES}

[1] Keller, U. "Recent developments in compact ultrafast lasers." Nature 424, no. 6950, 831-838 (2003).

[2] Okhotnikov, O.G., ed. [Semiconductor disk lasers: physics and technology], John Wiley \& Sons, (2010).

[3] Zewail, A.H., "Femtochemistry: Atomic-scale dynamics of the chemical bond.", J. Phys. Chem. A, 104(24), 5660-5694 (2000).

[4] Shah, J., [Ultrafast spectroscopy of semiconductors and semiconductor nanostructures], Vol. 115. Springer Science \& Business Media (2013).

[5] Valdmanis, J., and Mourou, G., "Subpicosecond electrooptic sampling: Principles and applications," IEEE J. Quantum Electron., 22(1), 69-78 (1986)

[6] Weingarten, K.J., Rodwel, M.J.W., and Bloom, D.M., "Picosecond optical sampling of GaAs integrated circuits," IEEE J. Quantum Electron., 24(2), 198-220 (1988).

[7] Miller, D.A.B., "Optical interconnects to silicon,” IEEE J. Sel. Top. Quantum Electron., 6(6), 1312-1317 (2000). 
[8] Mollenauer, L. F., Mamyshev, P.V., Gripp,J., Neubelt, M. J., Mamysheva, N., Grüner-Nielsen, L., and Veng, T., "Demonstration of massive wavelength-division multiplexing over transoceanic distances by use of dispersion-managed solitons," Opt. Lett., 25(10), 704-706 (2000).

[9]Krishnamoorthy, A.V., and Miller, D.A.B., "Scaling optoelectronic-VLSI circuits into the 21st century: A technology roadmap," IEEE J. Sel. Top. Quantum. Elecron., 2(1), 55-76 (1996).

[10] Huang, D., Swanson, E.A., Lin, C.P., Schuman, J.S., Stinson, W.G., Chang, W., Hee, M.R, Flotte, T., Gregory, K., Puliafito, C.A., Fujimoto, J.G., "Optical coherence tomography," Science (New York, NY), 254(5035), 1178 (1991).

[11] Fujimoto, J.G., "Optical coherence tomography," Comptes Rendus Acad. Sci., 2(8), 1099-1111 (2001).

[12] Marchese, S. V., Baer, C. R. E., Engqvist, A. G., Hashimoto, S., Maas, D. J. H. C., Golling, M., Südmeyer, T., and Keller, U., "Femtosecond thin disk laser oscillator with pulse energy beyond the 10-microjoule level." Opt. Express, 16(9), 6397-6407 (2008).

[13] Neuhaus, J., Bauer, D., Zhang, J., Killi, A., Kleinbauer, J., Kumkar,M., Weiler, S., Guina,M., Sutter, D.H., and Dekorsy, T., "Subpicosecond thin-disk laser oscillator with pulse energies of up to 25.9 microjoules by use of an active multipass geometry," Opt. Express 16(25), 20530-20539 (2008).

[14] Rafailov, E.U., [The physics and engineering of compact quantum dot-based lasers for biophotonics], John Wiley \& Sons, (2013). [15] Liu, X., Du, D., and Mourou, G., "Laser ablation and micromachining with ultrashort laser pulses," IEEE J. Quantum Electron., 33(10), 1706-1716 (1997).

[16] Juhasz, T., Loesel, F.H., Kurtz, R.M., Horvath, C., Bille, J.F., and Mourou,G., "Corneal refractive surgery with femtosecond lasers," IEEE J. Sel. Top. Quantum Electron., 5(4), 902-910 (1999).

[17] Hammer, D.X., Thomas, R.J., Noojin, G.D., Rockwell, B.A., Kennedy, P.K., and Roach, W.P, "Experimental investigation of ultrashort pulse laser-induced breakdown thresholds in aqueous media." IEEE J. Quantum Electron., 32(4), 670-678 (1996).

[18] Keller, U., Weingarten, K.J., Kartner, F.X., Kopf, D., Braun, B., Jung, I.D., Fluck, R., Honninger, C., Matuschek, N., and Aus Der $\mathrm{Au}$, J., "Semiconductor saturable absorber mirrors (SESAM's) for femtosecond to nanosecond pulse generation in solid-state lasers," IEEE J. Sel. Top. Quantum. Electon., 2(3), 435-453 (1996).

[19] Keller, U., Miller, D.A.B., Boyd, G.D., Chiu, T.H., Ferguson, J. F., and Asom, M. T., "Solid-state low-loss intracavity saturable absorber for Nd: YLF lasers: an antiresonant semiconductor Fabry-Perot saturable absorber." Opt. Lett., 17(7), $505-507$ (1992).

[20] Innerhofer, E., Südmeyer, T., Brunner, F., Häring, R., Aschwanden, A., Paschotta, R., Hönninger, C., Kumkar, M., and Keller, U., "60-W average power in 810-fs pulses from a thin-disk Yb: YAG laser," Opt. Lett., 28(5), 367-369 (2003).

[21] Brunner, F., Innerhofer, E., Marchese, S.V., Südmeyer, T., Paschotta, R., Usami, T., Ito, H., Kurimura, S., Kitamura, K., Arisholm, G., Keller, U., "Powerful red-green-blue laser source pumped with a mode-locked thin disk laser," Opt. Lett., 29(16), 1921-1923 (2004). [22] Hartl, I., McKay, H.A, Thapa, R., Thomas, B.K., Ruehl, A., Dong, L., and Fermann, M.E., "Fully stabilized GHz Yb-fiber laser frequency comb," In Advanced Solid-State Photonics, p. MF9. Optical Society of America, (2009).

[23] Lu, Z. G., Liu, J.R., Raymond, S., Poole, P.J., Barrios, P.J., and Poitras,D., "312-fs pulse generation from a passive C-band InAs/InP quantum dot mode-locked laser," Opt. Express, 16(14), 10835-10840 (2008).

[24] Plant, J.J., Gopinath, J.T, Chann, B., Ripin, D.J., Huang, R.K., and Juodawlkis, P.W., "250 mW, $1.5 \mu$ m monolithic passively modelocked slab-coupled optical waveguide laser," Opt. Lett., 31(2), 223-225 (2006).

[25] Rosales, R., Kalosha, V.P, Posilović, K., Miah, M.J., Bimberg, D., Pohl, J., and Weyers, M., "High brightness photonic band crystal semiconductor lasers in the passive mode locking regime," Appl. Phys. Lett., 105(16), 161101 (2014).

[26] Balzer, J. C., Schlauch, T., Klehr, A., Erbert, G., Tränkle, G., and Hofmann, M. R., "High peak power pulses from dispersion optimised modelocked semiconductor laser," Electron. Lett., 49(13), 838-839 (2013).

[27] Puchert, R., Bärwolff, A., Menzel, U., Lau, A., Voss, M., and Elsaesser,T., "Facet and bulk heating of GaAs/AlGaAs high-power laser arrays studied in spatially resolved emission and micro-Raman experiments," J. Appl. Phys., 80(10), 5559-5563 (1996).

[28] Laurain, A., Rockmore, R., Chan, H-T., Hader, J., Koch, S.W., Perez, A.R., Stolz, W., Moloney, J.V., "Pulse interactions in a colliding pulse mode-locked vertical external cavity surface emitting laser," J. Opt. Soc. Am. B, 34(2), 329-337 (2017).

[29] Saraceno, C.J., Schriber, C., Mangold, M., Hoffmann, M., Heckl, O.H., Baer, C.RE., Golling, M., Südmeyer, T., and Keller, U., "SESAMs for high-power oscillators: design guidelines and damage thresholds," IEEE J. Sel. Top. Quantum Electron., 18 (1), $29-41$ (2012).

[30] Grange, R., Haiml, M., Paschotta, R., Spühler, G.J., Krainer, L., Golling, M., Ostinelli, O., and Keller,U., "New regime of inverse saturable absorption for self-stabilizing passively mode-locked lasers," Appl. Phys. B, 80(2), 151-158 (2005).

[31] Saraceno, C.J., Heckl, O.H., Baer, C.R.E., Golling, M., Südmeyer, T., Beil, K., Kränkel, C., Petermann, K., Huber, G., and Keller, U., "SESAMs for high-power femtosecond modelocking: power scaling of an Yb: LuScO 3 thin disk laser to $23 \mathrm{~W}$ and $235 \mathrm{fs}$," Opt. Express., 19(2), 20288-20300 (2011).

[32] Baer, C.RE, Heckl, O.H., Saraceno, C.J., Schriber, C., Kränkel, C., Südmeyer, T., and Keller, U., "Frontiers in passively modelocked high-power thin disk laser oscillators," Opt. Express, 20(7), 7054-7065 (2012).

[33]Viskontas, K., Regelskis, K., and Rusteika, N., "Slow and fast optical degradation of the SESAM for fiber laser mode-locking at 1 $\mu \mathrm{m} . "$ Lith. J. Phys., 54(3), (2014).

[34] Diebold, A., Zengerle, T., Alfieri, C.G.E, Schriber, C., Emaury, F., Mangold, M., Hoffmann, M., Saraceno, C.J., Golling, M., Follman, D., Cole, G.D., Aspelmeyer, M., Sudmeyer, T., Keller, U., "Optimized SESAMs for kilowatt-level ultrafast lasers," Opt. Express, 24(10), 10512-10526 (2016).

[35] Haiml, M., Grange, R., and Keller,U., "Optical characterization of semiconductor saturable absorbers," Appl. Phys. B, 79 (3), 331339 (2004). 
[36] Alfieri, C. G. E., Diebold, A., Emaury, F., Gini, E., Saraceno, C.J., and Keller, U., "Improved SESAMs for femtosecond pulse generation approaching the kW average power regime," Opt. Express, 24 (24), 27587-27599 (2016).

[37] Kaiser, W., and Garrett, C.G.B., "Two-photon excitation in Ca F 2: Eu 2+," Phys. Rev. Lett., 7 (6), 229 (1961).

[38] Sin, Y., Presser, N., Foran, B., Ives, N., and Moss, S.C, "Catastrophic facet and bulk degradation in high power multi-mode InGaAs strained quantum well single emitters." SPIE LASE: Lasers and Applications in Science and Engineering, International Society for Optics and Photonics, 719818-719818 (2009).

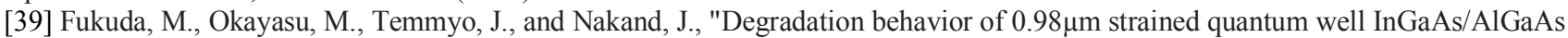
lasers under high-power operation," IEEE J. Quantum Electron., 30 (2), 471-476 (1994).

[40] Sin, Y., Presser, N., Foran, B., and Moss, S.C., "Investigation of catastrophic degradation in high power multi-mode InGaAs strained quantum well single emitters." In Lasers and Applications in Science and Engineering, International Society for Optics and Photonics, 68760R-68760R (2008).

[41] Okayasu, M., Fukuda, M., Takeshita, T., Uehara, S., and Kurumada, K., "Facet oxidation of InGaAs/GaAs strained quantum-well lasers," J. Appl. Phys., 69 (12), 8346-8351 (1991).

[42] Stryland, V., Eric W., Woodall, M.H., Vanherzeele, H., and Soileau, M.J., "Energy band-gap dependence of two-photon absorption," Opt. Lett., 10 (10), 490-492 (1985). 